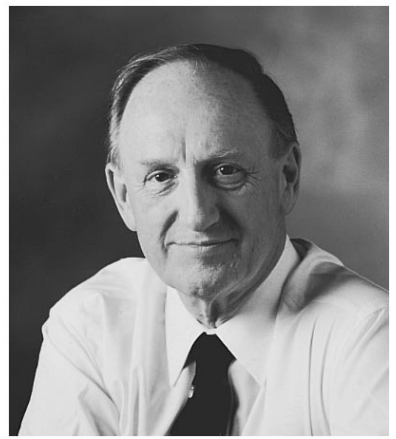

\section{Happy 80th Birthday Alexander Prokhorov}

I was approached at a recent meeting by Dr. Edmund I. Akopov to see if I would consider an editorial congratulating Dr. Alexander M. Prokhorov on his 80th birthday and acknowledge his many contributions to our field of optical science and engineering. While not working to create a precedent I was most happy to accept the request. In the expectation (hope?) that I might agree, Dr. Akopov provided me with the following details of Dr. Prokhorov's past and continuing scientific achievements.

Alexander M. Prokhorov, we salute you and your outstanding contributions as you celebrate your 80th birthday as an active and productive colleague.

\section{Alexander Prokhorov, Nobel Prize Winner}

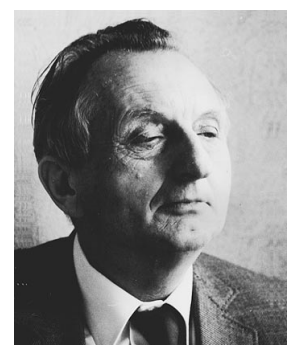

The famous Russian scientist Alexander M. Prokhorov was born in Atterton, Australia, on July 11, 1916. His scientific activity began in 1939 as a postgraduate of the Lebedev Physical Institute (LPI) of the USSR Academy of Sciences. Between 1942 and 1944, he took part in the Second World War where he served in a reconnaissance detachment. In 1944, after demobilization from wounding during active duty, Prokhorov came back to LPI, and was engaged in fundamental studies on the physics of oscillations, and, in particular, the theory of frequency stabilization of valve generators.

In the following years, he studied electron radiation in synchrotrons. It was demonstrated for the first time that a synchrotron could be used as a source of coherent elec- tromagnetic radiation in the centimeter spectral band. Now it is well known as synchrotron radiation. The results of these studies were the basis of Prokhorov's doctoral degree. Being the head of a group of young scientists in LPI, he began research in the novel area of modern physics-radio spectroscopy. This work prepared the background to the main ideas of quantum electronics.

In 1953, Prokhorov and N. G. Basov formulated the main principles for the theory of molecular oscillators and amplifiers. Then they proposed and experimentally realized a maser on ammonia molecules. In 1955, they suggested a method to create population inversions-a method of auxiliary radiation (optical pumping) in multilevel systems. It has resulted in the creation of a completely new class of low-noise radio receiving devicesmasers on paramagnetic crystals and then lasers. Prokhorov's work on microwave quantum amplifiers resulted in the creation of a complex of quantum paramagnetic amplifiers with extremely high sensitivity. His fundamental proposal for the development of quantum electronics was to use a new type of resonator-an open cavity formed by two parallel mirrors with the sizes and distance in between much larger than the radiation wavelength - the well-known Fabry-Pérot resonator.

A significant place in the research of Prokhorov was taken by his work on the creation of different types of efficient lasers based on various solid state media: crystals and glasses, powerful gas lasers, and lasers for technology, medicine, etc. Of great significance was his work in defining the upper limiting length of an active element of a laser, which limits the amplification of laser radiation with the increasing length of the active element. This conclusion turned out to be essential for the creation of powerful laser systems.

In 1966, he proposed and then realized a new type of a 
powerful gas laser, the gas dynamic laser with the direct transformation of thermal energy in coherent electromagnetic radiation. Around the mid-1960s, he also initiated and led work on a laser-produced high-temperature plasma, which promoted the development of a novel field of physics, laser thermonuclear fusion.

A broad range of research on the interaction of powerful laser radiation with gas and condensed matter has been conducted under Prokhorov's leadership. Many new physical effects, such as the multifocusing structure of a wave beam, propagating in nonlinear media; nonthermal resonant excitation of molecules by infrared radiation, resulting in their dissociation; laser generation of ultra sound in absorbing media; "slow burning" of a plasma in close vicinity of a target; and so on.

The leading positions in the science world have been taken by Prokhorov's results in submillimeter spectroscopy. At the beginning of the 1970s he initiated and promoted the development of novel technologies in Russia, such as integrated optics and optical communication by fiber waveguides.
In 1983, he organized the General Physics Institute (GPI) of the Russian Academy of Sciences, which he still successfully leads. Since then the GPI has become one of the world's leading institutions in the field of laser physics.

The fundamental research on solid state physics at the GPI under the management of Prokhorov promoted the establishment of new directions in the technology of materials-manufacturing of pure high-temperature materials, glasses, and ceramics. It has made a significant impact in energetics, metallurgy, optical communication, and quantum electronics.

In the last few years Prokhorov's leadership has been directed toward broad advanced research in environmental protection, laser medicine, and the physical backgrounds of perspective technologies. He has received many national and international awards and is a member of several academies and societies.

Brian J. Thompson Editor 